\title{
Statistical Features and Determinism in Partial Discharge Data from Electrical Trees and Voids
}

\author{
Nikola M. Chalashkanov, Conor P. Clarke, Stephen J. Dodd \\ School of Engineering, University of Lincoln, UK \\ nchalashkanov@lincoln.ac.uk
}

\begin{abstract}
Partial discharge (PD) data obtained from electrical trees and void experiments were analyzed. Several correlations were identified between the various statistical parameters traditionally used in PD classification and deterministic features such as average power dissipation. The correlation coefficients were found to have very high values in the case of electrical trees. Numerical simulations of PD activity in tree structures and voids were then used to explain the origin of the observed dependencies. The correlations between the statistical parameters describing the PD data identified in this work, could potentially be used to improve the robustness and reliability of defect classification based on PD measurements.
\end{abstract}

\section{INTRODUCTION}

Partial discharge is one of the most important degradation mechanisms in electrical insulation systems [1]. Internal partial discharges (PDs) in solid electrical insulation can lead to the formation of electrical trees [2]. The electrical trees propagate fast through the dielectric driven by the partial discharge activity in the existing tree channel structure. Therefore, PD activity is a valuable indicator for the extent of the electrical tree growth. The tree growth constitutes an accelerated degradation mechanism that can lead to insulation breakdown [3]. Evidence has shown that the PD activity driving electrical tree growth is governed by a deterministic mechanism with chaotic behavior [4,5] rather than being a purely stochastic process [6].

Partial discharge data are often acquired as phase resolved data, and analyzed statistically in terms of $\varphi-q-n$ patterns, where $\varphi_{i}$ is the phase of occurrence of the i-th discharge with respect to the applied ac voltage, $\mathrm{q}_{i}$ is the magnitude of the $i$-th discharge and $n$ is the number of discharges recorded in the acquisition window [7]. In order to characterize the data, various statistical parameters are calculated, which form the so called "fingerprint" of the defect causing the discharge activity [8].

Previous work [9] identified several correlations between the statistical parameters often used as defect fingerprints. Also, a correlation was found between the standard deviation of the total distribution of the discharge magnitudes (positive and negative) and the average power per discharge, which gives a quantitative relationship between the statistical and deterministic features of PD activity. If the PD power dissipated in the sample, is designated as $P$, then it can be calculated from the following equation:

$$
P=\frac{1}{\Delta t} \sum_{i=1}^{N} V_{i} q_{i}
$$

Where $\Delta t$ is the acquisition time, $V_{i}$ - instantaneous voltage at the occurrence of the i-th PD pulse, $q_{i^{-}}$magnitude of the i-th PD pulse, $N$ - number of PD pulses in the acquisition time $\Delta t$. The average power per discharge, $P_{a v}$, is calculated from the ratio of the dissipated power and the number of discharges:

$$
P_{a v}=P / N
$$

The standard deviation of the total distribution of the discharge magnitudes, $\sigma_{q}$, is calculated from (3):

$$
\sigma_{q}=\sqrt{\frac{\sum\left(q_{i}-\bar{q}\right)^{2}}{N}}=\sqrt{\frac{\sum q_{i}^{2}}{N}-\bar{q}^{2}}
$$

where $\bar{q}$ is the average value of the PDs in the acquisition period $\Delta t$. However, the total distribution of the discharge magnitudes is approximately a symmetrical distribution around zero, so the average charge is approximately equal to zero, $\bar{q} \approx 0$. Therefore, the second term to the right can be neglected in (3), and hence the expression for the standard deviation can be reduced to:

$$
\sigma_{q} \approx \sqrt{\frac{\sum q_{i}^{2}}{N}}
$$

From the correlation found in [9], the average power is proportional to the standard deviation $\sigma_{q}$.

$$
P_{a v} \propto \sigma_{q}
$$

If a constant of proportionality, $A$, is introduced in (5), it can be re-written as:

$$
P_{a v}=A \sigma_{q}
$$

Substituting (1), (2) and (4) into (6) and rearranging the two sides, gives the following expression relating $V_{i}, q_{i}$ and $N$ :

$$
\frac{\sum V_{i} q_{i}}{\sqrt{N \sum q_{i}^{2}}} \approx \text { const }
$$

Expression (7) was found to be valid for the whole set of applied voltages studied in [9]. The value of the constant was found to be determined by the magnitude of applied test voltage.

The work reported here aims to build on the observed correlations detailed above of the statistical features of PD patterns with deterministic aspects of PD such as power 
dissipation. The focus of this work is on PD data obtained from electrical trees and electrode-bound voids in epoxy resins and will be supported by physical modelling of the PD activity through computer simulations.

\section{EXPERIMENTAL}

\section{A. Sample Preparation}

Samples were prepared from two DGBEA epoxy resin systems, namely Araldite CY1301 and Araldite CY1311. The latter resin is a modified version of the former with added plasticizer. When fully cured, the glass transition temperature, $\boldsymbol{T}_{\boldsymbol{g}}$, of CY1301 is approximately $50{ }^{\circ} \mathrm{C}$, while $\boldsymbol{T}_{\boldsymbol{g}}$ of CY1311 is $0{ }^{\circ} \mathrm{C}$, and hence it is in a flexible state at room temperature. The test samples suitable for electrical tree growth experiments were manufactured using tungsten carbide pins of $1 \mathrm{~mm}$ diameter and pin-tip radius of $3 \mu \mathrm{m}$, which were cast in either Araldite CY1311 epoxy slabs. The distance between the pin and the plane was $2 \pm 0.5 \mathrm{~mm}$. The samples were tested electrically 10-12 weeks after their manufacture (post cure stage).

Araldite CY1301 samples were used for the void samples due to its greater rigidity. These were produced in the same way as the treeing samples by partially extracting the pin following the post cure stage and thus creating a void with a length, $\boldsymbol{l}_{v}$, of $1 \pm 0.3 \mathrm{~mm}$, as shown in Fig. 1. A hydraulic jig was used to extract the pins. However, the extraction procedure was not perfect and caused de-bonding of the pin from the epoxy matrix and some damage (cracking) to the resin near to the pin-tip.

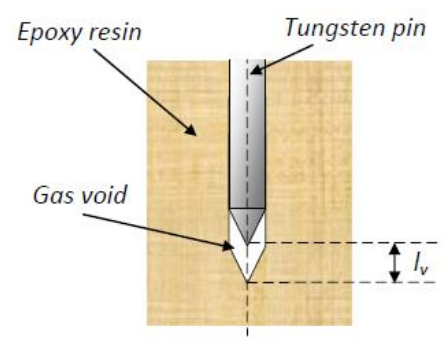

Fig. 1. Void geometry

Following the partial extraction and to prevent subsequent movement of the pin, a two-part rapid cure epoxy adhesive was used to bond the pin to the top of the sample. In this way, the void formed in the sample was also sealed from the environment. All samples (treeing and void) were stored in humidity-controlled containers providing a range of relative humidity between $15 \%$ and $100 \%$. All samples were periodically weighted with a precision balance. The amount of absorbed moisture was characterized for each sample by determination of the percentage mass uptake, $\Delta \mathrm{M} \%$, from the reference weight measured immediately after the post-cure.

\section{B. Electrical Tests}

The electrical tests were performed in a Faraday cage to minimize the external interference. A RLC detector with a resonant frequency of $200 \mathrm{kHz}$ was used. The detector was linked to a digital storage oscilloscope via an optical link to prevent external interference and to protect the oscilloscope in a case of sample breakdown. The PD detector and the measurement system were calibrated using a capacitor of 10 $\mathrm{pF}$ and a square wave generator of $10 \mathrm{~V}$ amplitude to provide an injecting charge of $100 \mathrm{pC}$. The noise level of the PD detection system was $\pm 1.5 \mathrm{pC}$. The HV transformer has been found to be discharge free below $17 \mathrm{kV}$ rms. The test sample was contained within a temperature-controlled oil bath to allow experiments to be conducted at temperatures between 20 ${ }^{\circ} \mathrm{C}$ and $60{ }^{\circ} \mathrm{C}$. Further details of the experimental protocol are given in [10]. The data acquisition time was $1 \mathrm{~s}$, repeated every $10 \mathrm{~s}$ throughout the test. The electrical trees were grown at applied ac voltage of $13.5 \mathrm{kV}$ rms. The void tests were conducted with applied ac voltage of $7.5 \mathrm{kV}$ rms.

\section{PD SIMULATIONS}

Two different computer based numerical models were used to simulate the PD activity in either electrical trees or in electrode-bound voids. The simulated partial discharges within electrically non-conducting tree structures were based on the deterministic modelling approach described in [11, 12]. The simulations were based on using spheres of charge to describe the pin electrode and the induced charge at the pin-tip from charge contained within the tree structure. Charge dipoles were added to the tree structure to simulate the occurrence of local electron avalanches. The conditions for a local electron avalanche to occur at a particular time step was that the tree segment potential drop must exceed a value $\mathrm{V}_{\text {on }}$, called the inception voltage. Dipoles of charge were added until the potential drop along each tree segment was less than the extinction voltage, $\mathrm{V}_{\text {off. }}$. Each partial discharge event was therefore the sum of one or more local electron avalanches occurring within the tree structure at a particular time step. The apparent charge was calculated from the change in the calculated induced charge on the pin electrode following each discharge event. The charge representing the pin potential was assumed to vary sinusoidally with a frequency of $50 \mathrm{~Hz}$. Tree channel conductivity was simulated by calculating the charge flow along each tree segment in proportion to the segment potential difference. Thus, the conduction of charge along tree segments was considered Ohmic. Fig. 2 shows an example of a model tree structure.

MATLAB was used to simulate partial discharge activity in voids. Fig. 3 shows a simplified equivalent circuit model that was used to study the effect of bulk charge transport on the PDs. The circuit represents a void in series with the bulk of the material. 


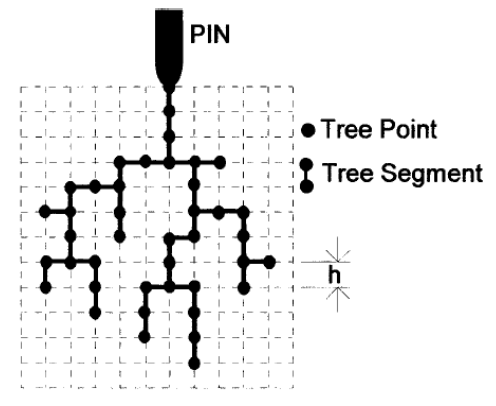

Fig. 2. Electrical tree model

The void is represented as a spark gap in parallel with $G_{v}$, where $G_{V}$ is the surface conductance of the void. A capacitor $C_{B}$ in parallel with a conductance $G_{B}$ represents the bulk capacitance and conductance of the epoxy resin, respectively. This model was used for simulation of the PD activity in the case of an electrode-bound voids. The electric field for PD inception, $\mathrm{E}_{\mathrm{on}}$, and extinction, $\mathrm{E}_{\text {off }}$, were separately defined for each half-cycle of the applied voltage stress, $\mathrm{E}_{\mathrm{AC}}$, as free parameters. The values of $E_{\text {on }}$ and $E_{\text {off }}$ were chosen to match typical experimental PD data and had similar values for both polarities as the PD discharge patterns are essentially symmetric. In order to obtain variability in the magnitudes of the simulated discharges, it was necessary to add a small time dependent random generated component to the values of $E_{\text {on }}$ and $\mathrm{E}_{\text {off. }}$ Therefore, the model can be considered as pseudodeterministic. The value of value of $\mathrm{G}_{\mathrm{V}}$ was set to zero to model a void wall that is non-conducting and the value of $C_{B}$ was chosen to be $2.2 \mathrm{nF}$.

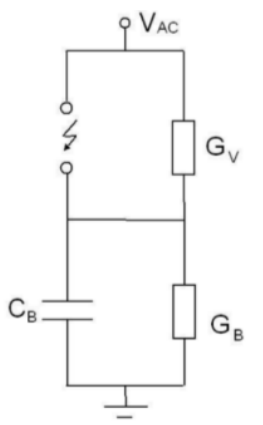

Fig. 3. Equivalent circuit for PD simulation in voids

\section{RESUlTS AND DISCUSSION}

\section{A. Electrical trees}

A typical plot of the average power of the discharge plotted against the standard deviation of the PD amplitudes during the growth of an electrical tree is shown in Fig. 4. Each point represents the average PD power plotted against the standard deviation of the PD amplitudes acquired at each one second acquisition. The plot indicates very strong linear correlation between the two quantities over the duration of the tree growth experiment. In fact, this strong correlation, with correlation coefficients approaching a value of 1 , between the average power of the discharges and the standard deviation of their amplitudes was observed in all data sets obtained from electrical treeing experiments, see Table I. Previously, the same dependence was discovered in the PD data from electrical trees grown at different voltages [9].

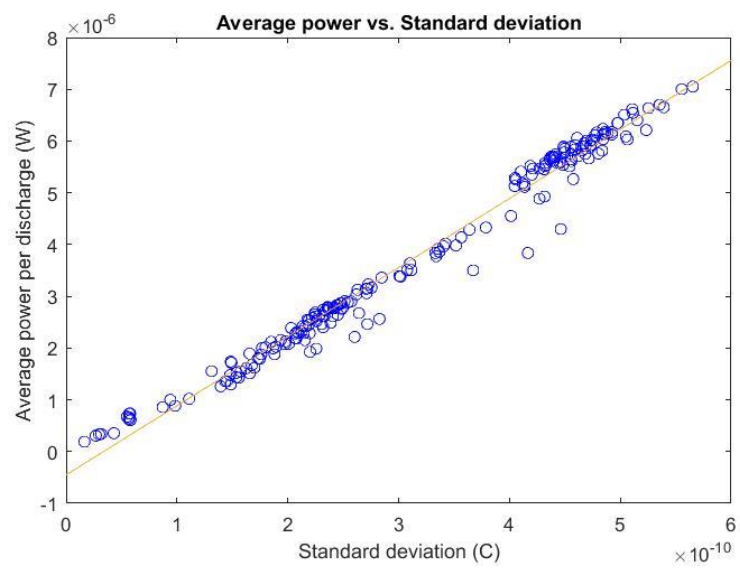

Fig. 4. Plot of the average power of the PDs vs the standard deviation of their amplitudes (experimental data)

TABLE I

Correlation Coefficient between Average Discharge Power and the Standard Deviation of Discharge Magnitudes. Data From Electrical Trees Grown In ARALDITE CY1311 EpoXy Resin SAMPLES

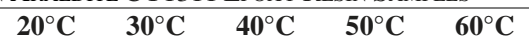

\begin{tabular}{lllllc}
\hline Mass uptake 0.1\% & 0.9908 & 0.9777 & 0.9911 & 0.9983 & 0.9991 \\
\hline Mass uptake 0.6\% & 0.9916 & 0.9931 & 0.9943 & - & -
\end{tabular}

Mass uptake 1.0\% $\quad 0.9777 \quad 0.9944$

The shape of the electrical trees grown (often characterized by the fractal dimension, $\mathrm{d}_{\mathrm{f}}$ ) depended on the environmental conditions. Bush trees $\left(2<\mathrm{d}_{\mathrm{f}}<3\right)$ were observed at low moisture uptake $(0.1 \%)$ and temperatures $\left(20{ }^{\circ} \mathrm{C}\right)$, while increasing the moisture content and/or the test temperature led to reduction of the fractal dimension of the tree structure, and hence branch trees $\left(1<\mathrm{d}_{\mathrm{f}}<2\right)$ were observed at higher moisture uptake and test temperatures [10]. Despite the significant changes in the fractal dimension, the correlation coefficient between the standard deviation of the PD amplitudes and the average power remained very high in all cases, which is an indication that the observed correlation is independent of the fractal dimension of the tree structures.

In order to replicate the observed strong correlation between PD average power and PD standard deviation, a set of PD data were simulated using the numerical modelling approach described in $[11,12]$. The tree growth was emulated by starting with a numerical simulation of PD activity with one tree segment at the pin tip. Following this, successive tree segments were added to the tree structure one at a time followed by a repeat simulation. The position of the new tree segment was chosen to mimic the growth of a real tree. Each 
simulation represented the PD activity in the tree structure over $1 \mathrm{~s}$ to match the data acquisition period of the experimental data. In total 40 simulation files were generated, each file containing phase resolved $\mathrm{PD}$ data. The final electrical tree structure containing all 40 tree segments used in the simulations is shown in Fig. 5

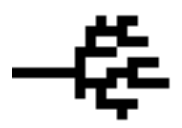

Fig. 5. Tree structure used for the PD simulations

The plot of the standard deviation of the simulated PD amplitudes against the average power of the simulated discharges is shown in Fig.6. The data shows an approximate straight line over the time of tree growth. The correlation coefficient obtained from the simulated data was 0.9965 . The numerical simulation results therefore showed very good agreement with the experiments.

The very high values of the correlation coefficient obtained from all experimental and simulated data indicate that the relationship between the average discharge power and the standard deviation of the discharge magnitudes is characteristic the electrical tree growth process and provides evidence for the deterministic origin of this process.

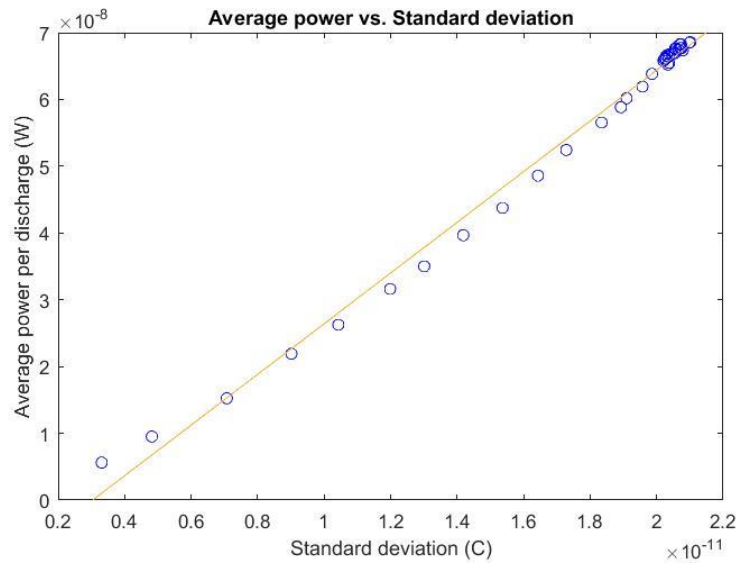

Fig. 6. Plot of the average power of the PDs vs the standard deviation of their amplitudes (simulated data)

\section{B. Partial discharges in voids}

The partial discharge data obtained from voids in Araldite CY1311 and Araldite CY1301 epoxy resin samples was analyzed using the same procedure as described above. The corresponding correlations coefficients are given in Tables II and Table III, respectively for the two epoxy resin types. In the case of the void samples, there is much more variation in the values of the correlation coefficient between the average power of the discharges and the standard deviation of their amplitudes. Some data sets indicate good correlation whilst others show little correlation.

The values of the correlation coefficient in Table II and Table III present a complicated picture. There is no simple dependence of the correlation coefficient values on either temperature or absorbed moisture. There are number of other factors, which could account for the variability observed in the data. For example, the void length was difficult to control and not all samples are identical in this regard. Furthermore, the surface of the void obtained as a result of the partial extraction of the pin was not always perfect (see Fig. 1). In some cases, small cracks could have been formed during the pin extraction, which could have provided additional channels for partial discharges to take place and thus created a fractal system akin of an electrical tree. This may account for the very high correlation coefficient in some void samples of both CY1311 and CY1301 resins.

TABLE II

Correlation Coefficient Between Average Discharge Power and the Standard Deviation of Discharge Magnitudes. Data from Samples with VoIDS IN ARALDITE CY1311 EpoXy RESIN.

\begin{tabular}{lcccc}
\hline & $\mathbf{2 0}^{\circ} \mathbf{C}$ & $\mathbf{3 0}^{\circ} \mathbf{C}$ & $\mathbf{4 0}^{\circ} \mathbf{C}$ & $\mathbf{5 0}^{\circ} \mathbf{C}$ \\
\hline Mass uptake 0.1\% & 0.9910 & - & - & - \\
\hline Mass uptake 0.7\% & 0.2304 & 0.6053 & 0.9181 & 0.7258 \\
\hline Mass uptake 1.1\% & 0.5026 & - & - & - \\
\hline Mass uptake 2.5\% & 0.9526 & - & - & -
\end{tabular}

TABLE III

Correlation Coefficient Between Average Discharge Power and the Standard Deviation of Discharge Magnitudes. Data From Samples with VOIDS IN ARALDITE CY1301 EPOXY RESIN

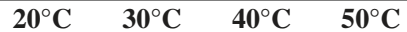

\begin{tabular}{lcllll}
\hline Mass uptake 0.1\% & 0.9885 & - & - & - \\
\hline Mass uptake 0.2\% & -0.1172 & 0.9878 & 0.4764 & 0.9975 \\
\hline Mass uptake 0.3\% & 0.1947 & - & - & - \\
\hline Mass uptake 0.6\% & 0.3393 & - & - & -
\end{tabular}

Another important difference between the two epoxy resin systems was their glass transition temperature, $\mathrm{T}_{\mathrm{g}}$, which determines the dielectric properties of each epoxy system [13]. It has been found in [13] that above the glass transition temperature of the resin, the long-range charge transport processes are both temperature and absorbed moisture dependent. In order to investigate how the bulk material conductivity affects the PD activity in voids and hence its effect on the correlation coefficient between the average discharge power and the standard deviation of their magnitudes, a simple equivalent circuit model was used (see Fig.3). Ten files were simulated for each value of $G_{b}$. Each file represents PD activity in a void over a period of $1 \mathrm{~s}$. The values of the average power and the standard deviation of the discharge amplitudes were calculated for each file and hence the correlation coefficient was obtained. The results are summarized in Table IV. From Table IV, increasing the bulk conductivity of the material tends to decrease the value of the correlation coefficient. 
The increased bulk conductivity removes the space charge deposited by a previous discharge and thus increases the possibility for further discharges to take place at the same half-cycle of the applied voltage [10]. As a result, there is a significant increase in the number of PDs and their phases of occurrence tend to shift and align with the applied voltage. On the other hand, the average PD amplitudes decrease. These changes in the PD statistics, perhaps, account for the lower correlation coefficient in the middle of the conductivity range studied here. At the highest value of the bulk conductivity given in Table IV, the value of the correlation coefficient increases again. It is interesting to note that the results from the experimental data given in Table II and Table III roughly follow the same pattern. Hence, changes in the bulk material conductivity could explain the observed fluctuations in the value of the correlation coefficient.

TABLE IV

Dependence of the CorRelation Coefficient on the Value of the Modelling Parameter $\mathrm{G}_{\mathrm{B}}$, Representing the Bulk Conductivity of the

\begin{tabular}{cc}
\multicolumn{2}{c}{ RESIN } \\
\hline $\mathbf{G}_{\mathbf{b}}[\mathbf{S}]$ & Correlation Coefficient \\
\hline $5 \times 10^{-6}$ & 0.895618 \\
\hline $5 \times 10^{-7}$ & -0.24646 \\
\hline $5 \times 10^{-8}$ & 0.5002 \\
\hline $5 \times 10^{-9}$ & -0.36127 \\
\hline $5 \times 10^{-10}$ & 0.832493 \\
\hline $5 \times 10^{-11}$ & 0.824424 \\
\hline
\end{tabular}

\section{CONCLUSIONS}

The correlation coefficient between the average discharge power and the standard deviation of discharge magnitudes presents an intriguing indicator, which potentially can be used for discrimination between PDs from electrical trees and those occurring in voids. In all cases involving electrical trees, the value of the correlation coefficient was found to be very high, e.g. above 0.9 indicating a deterministic origin for the PD fluctuations. In the case of electrode-bound voids, the value of the correlation coefficient was found to vary significantly between the different test samples. This may indicate the increasing stochastic influence on PD activity. Factors that may affect its value involve the shape of the void, the uniformity of the void surface, and the electrical conductivity of the bulk dielectric material, which in turn depends on the level of absorbed moisture in the sample and the test temperature. Further work is required to clarify the relative importance of each factor.

\section{REFERENCES}

[1] L.A. Dissado and J.C. Fothergill, Electrical Degradation and Breakdown in Polymers, ed. G.C. Stevens, Peter Peregrinus Ltd., 1992
[2] L.A. Dissado, "Understanding Electrical Trees in Solids: From Experiment to Theory", IEEE Trans. Dielectrics and Electrical Insulation, Vol. 9, pp. 483-97, 2002

[3] S.W. Rowe, "Electrical Ageing of Composites: An Industrial Perspective", International Conference on Solid Dielectrics, Winchester, UK, July 8-13, 2007, pp 401-6

[4] S.J. Dodd, L.A.D., J.V. Champion, J.M. Alison, "Evidence for deterministic chaos as the origin of electrical tree breakdown structures in polymeric insulation", Physical Review B, Vol. 52(24), pp. R16 985R16 988, 1995.

[5] L.A. Dissado, J.C. Fothergill, N. Wise, A. Willby, J. Cooper, "A deterministic model for branched structures in the electrical breakdown of solid polymeric dielectrics", J. Phys. D: Appl. Phys., Vol. 33, pp. L109-L112, 2000.

[6] R. Altenburger, C. Heitz and J. Timmer,"Analysis of phase-resolved partial discharge patterns of voids based on a stochastic process approach", J. Phys. D: Appl. Phys., Vol. 35, pp. 1149-63, 2002

[7] N. C. Sahoo, M. M. A. Salama, and R. Bartnikas, "Trends in Partial Discharge Pattern Classification: A Survey," IEEE Trans. Dielectrics and Electrical Insulation, vol. 12, pp. 248-264, 2005.

[8] F.H. Kreuger, E. Gulski, and A. Krivda, "Classification of Partial Discharges," IEEE Trans. Electrical Insulation, vol. 28, pp. 917-931, 1993

[9] S.J. Dodd, N.M. Chalashkanov and J.C. Fothergill, "Statistical Analysis of Partial Discharges from Electrical Trees Grown in a Flexible Epoxy Resin”, 2008 Annual Report Conference on Electrical Insulation and Dielectric Phenomena, 26-29 Oct. 2008.

[10] N.M .Chalashkanov., S.J. Dodd, L.A. Dissado, J.C. Fothergill, "The Role of Bulk Charge Transport Processes in Electrical Tree Formation and Breakdown Mechanisms in Epoxy Resins", IEEE Trans. on Dielectrics and Electrical Insulation, Vol. 23 (6), 2016, p. 3256 - 3266

[11] J.V. Champion and S.J. Dodd, "An approach to the modelling of partial discharges in electrical trees", J. Phys. D: Appl. Phys., Vol. 31, pp. 2305-2314, 1998

[12] J.V. Champion and S.J. Dodd, "Simulation of partial discharges in conducting and non-conducting electrical tree structures", J. Phys. D: Appl. Phys., Vol. 34, pp. 1235-1242, 2001

[13] N.M. Chalashkanov, S.J. Dodd, L.A. Dissado, J.C. Fothergill, "Reexamination of the Dielectric Spectra of Epoxy Resins: Bulk Charge Transport and Interfacial Polarization Peaks", IEEE Trans. on Dielectrics and Electrical Insulation, Vol. 21 (3), pp. 1330-1341, 2014 